\title{
ARC DETECTION IN DC ARC FURNACES
}

\author{
Quinn G Reynolds ${ }^{1}$, Christopher J Hockaday ${ }^{1}$, Dominic T Jordan ${ }^{1}$, Ian J Barker ${ }^{2}$ \\ ${ }^{1}$ Mintek; Private Bag X3015; Randburg 2125; South Africa \\ ${ }^{2}$ Private Consultant; c/o Mintek; Private Bag X3015; Randburg 2125; South Africa
}

Keywords: Furnace, Plasma Arc, Control, Measurement, Modelling

\begin{abstract}
A direct-current (DC) plasma arc furnace is a type of electric furnace used for metallurgical operations. The successful operation of DC furnaces depends to a large extent on gaining a fundamental understanding of the arc phenomenon itself, and ensuring its presence in the furnace at all times. A method for detection of the presence of the arc in a DC circuit is presented, along with discussion of why this may be of value for certain modes of furnace operation such as brush-arc. The theoretical development of the method is presented along with supporting experimental work conducted on large-scale pilot-plant facilities.
\end{abstract}

\section{Introduction}

Direct-current (DC) plasma arc furnaces have seen widespread usage in the steel industry as a scrap remelting unit. More recently, process applications for DC furnaces in the smelting of primary ores have been gaining ground in industry. These applications include the smelting of chromite to produce ferrochromium, the smelting of ilmenite to produce titania slag and pig iron, and the smelting of nickel laterite to produce ferronickel [1].

A typical DC furnace consists of a cylindrical shell topped with a conical roof, through which the ore and chemical reagents needed for the process are fed. This vessel is lined with refractory material and may also include additional cooling systems such as forced-convection copper cooling elements. The vessel typically contains an open molten bath consisting of several different phases, frequently a light slag phase above a denser metal phase. One or more electrodes made of pre-baked graphite serve as cathodes, and enter through the center of the roof. The base of the furnace is designed with an electrically conductive anode in direct contact with the molten bath. The plasma arc exists between the tip of the electrode and the surface of the bath, and completes the circuit between the graphite electrode and the anode connection. The electrode and anode are connected to a DC power supply, which generally consists of a transformer unit, a rectifier unit, and one or more chokes. The transformer adjusts alternatingcurrent power from the grid to the appropriate voltages and currents, and the rectifier then converts the alternating current into DC. The chokes provide inductive capacity in order to damp any large spikes in voltage resulting from fluctuations in the furnace operation.

The arc is the primary heating and stirring element in the DC furnace, converting electrical energy into thermal energy and transferring it into the molten bath. It is a high-temperature, highvelocity jet consisting of plasma - material in the fourth state of matter. The thermal plasma in the arc is formed by the dissociation of the gases in the furnace freeboard region when they are heated to very high temperatures (above $5000 \mathrm{~K}$ ) and break down into a mixture of positive ions and negative electrons. Due to the presence of free electrons, the arc plasma conducts electricity effectively. 
Since the arc and slag bath are connected in series in the DC electric circuit through the furnace, part of the total operating voltage (and hence power dissipation) is split across each. The proportion in which the voltage is divided between the two is determined by the physical properties of the arc plasma and slag, as well as the geometry of the furnace [2]. In particular, for processes involving slags of high electrical resistivity (for example, in ferronickel production where slags have a moderate to high $\mathrm{SiO}_{2}$ content), the voltage drop across the slag layer is likely to be significant. This can result in either a high total voltage requirement for the furnace, or a limited voltage available for the arc with the majority of the voltage drop occurring across the slag. Very high voltage specifications can add considerable expense to the design of the DC power supply. The preference may therefore be to operate such a furnace with a reduced arc length, thereby limiting the total voltage while retaining the process advantages [1] of operating with an arc present. This brush-arc mode of operation has been applied in AC submerged arc furnace operations [3], and is likely to see application on DC plants in the future.

In addition to the expansion of DC applications into the smelting of high-resistivity materials, there is growing interest in the viability of retrofitting three-electrode circular AC furnaces to operate using DC power. Due to the cost and complexity involved in redesigning the hearth to include a bottom anode, a potentially attractive means of accomplishing the switch to DC is to use a dual-electrode design [4], with two separate graphite electrodes serving as anode and cathode respectively. In such a design, there are two arcs present inside the furnace vessel, and current passes through them in series via the slag layer. This can result in a significantly higher voltage requirement (approximately double) at a given current than a traditional single-electrode DC furnace, and again the preference may be to operate such furnaces with short arc lengths, in brush-arc mode [4].

Since many of the advantages of DC furnace operation are related to the presence of an open arc in the furnace, a means of determining when this is the case would be of some value. This is especially true for processes requiring brush-arc operation, in which it may be very difficult for operators to discern between open-arc (with the electrode tip a small distance above the slag) and resistance-heating (with the electrode tip in direct contact with the slag) conditions without visual inspection of the interior of the furnace during operation. In addition to being a safety hazard, this may be difficult or impossible depending on the design of the vessel.

\section{Theory of arc behaviour}

The DC plasma arc is a complex coupled system resulting from strong interactions between fluid flow, thermal, and electromagnetic fields inside the furnace vessel [5]. Due to the large velocities and driving forces involved, the plasma arc exhibits dynamic behaviour and changes shape rapidly on very short time scales, typically of the order of milliseconds or less [5]. Signals of interest to arc measurement studies are therefore expected to occur in the $\mathrm{kHz}$ and higher frequency range.

\section{The arc as a magneto-hydrodynamic (MHD) system}

Arcs may be described using three key physical aspects: a momentum transfer model, a heat transfer model, and an electromagnetic field model. Together these form an MHD system. A number of approximations are applied to the governing equations in order to simplify the mathematical description in the case of arcs consisting of thermal plasma. The MHD equations commonly used to model plasma arcs are presented below - more detail on the derivation of these equations and the approximations involved can be found in other publications [5].

The Navier-Stokes (1) and continuity (2) equations govern fluid flow for Newtonian fluids. 


$$
\begin{gathered}
\rho \frac{\partial \mathbf{v}}{\partial t}+(\rho \mathbf{v} \cdot \nabla) \mathbf{v}+\nabla P=\nabla \cdot(\mu \nabla \mathbf{v})+\mathbf{j} \times \mathbf{B} \\
\frac{\partial \rho}{\partial t}+\nabla \cdot(\rho \mathbf{v})=0
\end{gathered}
$$

Here, $\mathbf{v}$ is the velocity vector field, $\rho$ is the plasma fluid density, $\mathrm{P}$ is the pressure, and $\mu$ is the dynamic viscosity. $\mathbf{B}$ and $\mathbf{j}$ are the magnetic field intensity and the current density vector fields, which together produce the Lorentz force source term in (1) - this acts on the plasma to compress and accelerate it, resulting in the formation of an arc jet.

The temperature field is described by the energy conservation equation (3).

$$
\rho C_{P} \frac{\partial T}{\partial t}+\left(\rho C_{P} \mathbf{v} \cdot \nabla\right) T=\nabla \cdot(\kappa \nabla T)+\frac{\mathbf{j} \cdot \mathbf{j}}{\sigma}-Q_{R}
$$

Here, $\mathrm{T}$ is the plasma temperature, $\mathrm{C}_{\mathrm{P}}$ is the heat capacity, and $\kappa$ is the thermal conductivity. $\sigma$ is the electrical conductivity, and $Q_{R}$ is the volumetric rate of energy loss from the plasma by radiation. $Q_{R}$ and $\sigma$ are both strong functions of temperature. The last two terms on the righthand side of (3) constitute the energy source term for the plasma - energy is generated by the passage of electric current (Ohmic heating), and lost by thermal radiation.

The electromagnetic fields are given by the electrostatic and magnetostatic approximations to Maxwell's equations (4)-(6).

$$
\begin{gathered}
\mathbf{j}=-\sigma \nabla \phi \\
\nabla \cdot \mathbf{j}=0 \\
\nabla \times \mathbf{B}=\mu_{0} \mathbf{j}
\end{gathered}
$$

Here, $\phi$ is the electric potential (voltage) field, and $\mu_{0}$ is the permeability of free space, a physical constant.

This coupled set of equations governs $\mathbf{v}, \mathrm{T}, \mathbf{j}$, and $\mathbf{B}$ as they evolve in time to produce a selfsustained plasma arc.

\section{The arc as an inertial system}

It is reasonable to assume that the spatial shape of the arc jet, as determined by its temperature distribution, at any given time is approximately proportional to its electrical resistance (and therefore voltage at a fixed current). This is due to the fact that the temperature distribution strongly influences the electric potential distribution, via the temperature dependence of $\sigma$ in (4). Also, since the velocities in the arc are generally very high, convective energy transport is dominant in (3) and regions of high velocity tend to correlate with regions of high temperature as energy from the hot arc root near the electrode tip is carried down the jet into the body of the arc.

In order to gain an understanding of the dynamic behaviour of arc voltage, it is therefore of interest to examine how the momentum transport equation, (1), affects the arc jet's shape. Much of the high-velocity flow and rapid dynamic motion of arcs originates in regions where the $\mathbf{j} \times \mathbf{B}$ Lorentz force term in (1) is dominant. (1) may therefore be roughly approximated as:

$$
\rho \frac{\partial \mathbf{v}}{\partial t} \approx \mathbf{j} \times \mathbf{B} \equiv \mathbf{L}_{\mathbf{F}}
$$


Defining $\mathbf{v}$ as the first derivative in time of a displacement vector $\mathbf{r}$, and simplifying and rearranging (7), gives:

$$
\frac{\partial^{2} \mathbf{r}}{\partial t^{2}} \approx \frac{\mathbf{L}_{\mathbf{F}}}{\rho}
$$

Here, $\mathbf{L}_{\mathbf{F}}$ is the Lorentz force term, and $\mathbf{r}$ indicates how the shape of the arc changes in space with velocity field $\mathbf{v}$. $\mathbf{r}$ therefore directly affects the voltage and related external measurable parameters of the arc, and may be taken as a proxy for them in the analysis that follows.

(8) is seen to describe a simple inertial response system, in which a measured dependent variable (r) is affected by an independent control variable $\left(\mathbf{L}_{\mathbf{F}}\right)$ via the second derivative in time. It is interesting to consider the frequency response of such systems - if the Fourier transform of (8) is taken, we obtain:

$$
-(2 \pi \omega)^{2} \mathcal{F}(\mathbf{r})=\frac{\mathcal{F}\left(\mathbf{L}_{\mathbf{F}}\right)}{\rho}
$$

Considering the magnitude of the terms on either side of the equation, rearranging, and taking the logarithm then gives:

$$
\log _{10}|\mathcal{F}(\mathbf{r})|=\log _{10}\left[\frac{\left|\mathcal{F}\left(\mathbf{L}_{\mathbf{F}}\right)\right|}{4 \pi^{2} \rho}\right]-2 \log _{10} \omega
$$

If a white noise perturbation signal is assumed for $\mathbf{L}_{\mathbf{F}}$, consistent with random noise on the current input to the arc (which determines both $\mathbf{j}$ and $\mathbf{B}$ ), the first term on the right-hand side of (10) becomes constant. Plotting the magnitude of the Fourier transform of $\mathbf{r}$ against frequency $\omega$ on a log-log graph would then give a straight line with a gradient of -2 , or a fall-off of two decades per decade, as illustrated in Figure 1.
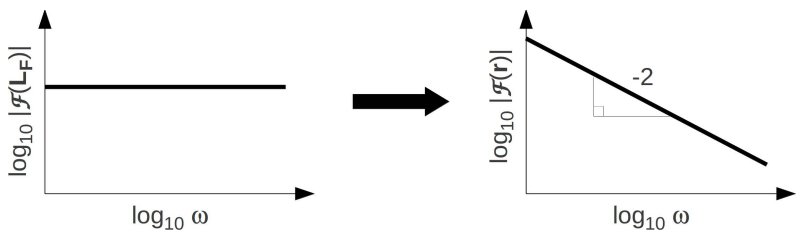

Figure 1: Signal analysis of a simplified inertial response system.

As $\mathbf{r}$ directly affects voltage and other measurable parameters of the arc in the present model, it is reasonable to propose that arcs may be identified from voltage signals measured on DC furnaces by performing a Fourier transform operation on the data and seeking parts of the spectrum that exhibit a two decades per decade falloff.

\section{Computational modelling of the arc}

A high resolution, three-dimensional computational model of a DC plasma arc in air was developed using numerical solutions of (1)-(6) [5]. The model calculates both the spatial and temporal development of the arc from specified initial and boundary conditions. The plasma gas used in the model was air, which determines the physical properties and temperature dependence of electrical conductivity and radiation energy loss. Other parameters used in the model are shown in Table I.

The model uses a box-shaped domain, with the upper surface adjacent to the tip of the electrode (which forms the cathode), and the bottom surface adjacent to the molten bath (which forms the 
anode). Constant temperatures and zero velocities are assumed at the electrode, bath and other boundary surfaces. An arc attachment zone is defined at the centre of the electrode tip, on which the current density is specified as $\mathrm{j}_{\mathrm{k}}=3.5 \mathrm{kA} / \mathrm{cm}^{2}$ [6]. This zone forms the root of the arc, which is established in the space between the electrode and bath.

Table I: Simulation parameters used in computational model of the arc.

\begin{tabular}{|l|l||l|l|}
\hline Parameter & Value & Parameter & Value \\
\hline Region dimensions & $0.1 \times 0.1 \times 0.05 \mathrm{~m}$ & Arc current & $500 \mathrm{~A}$ \\
\hline Electrode diameter & $0.05 \mathrm{~m}$ & Simulation time & $10 \mathrm{~ms}$ \\
\hline Arc root diameter & $0.00426 \mathrm{~m}$ & Mesh resolution & $384 \times 384 \times 192$ \\
\hline FFT sample size & 25000 & FFT time step size & $0.2 \mu \mathrm{s}$ \\
\hline
\end{tabular}

Operating under perfect DC conditions, the plasma arc model exhibits a large degree of highfrequency chaotic behaviour resulting from various fluid-dynamic and electromagnetic instabilities characteristic of plasma systems. This can be seen in plots of the temperature field at various times shown in Figure 2.

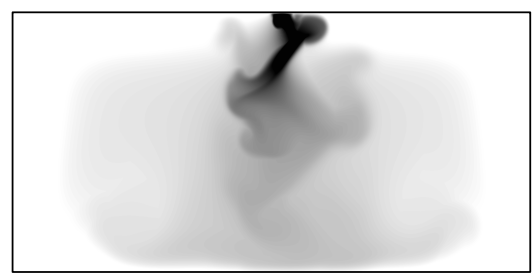

(a) $2.962 \mathrm{~ms}$

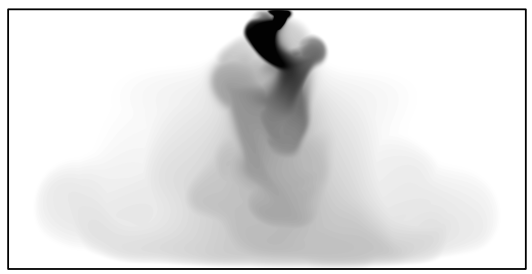

(b) $4.871 \mathrm{~ms}$

Figure 2: X-Z projections of temperature field from plasma arc model at various times, scale $5000 \mathrm{~K}$ (white) to $12000 \mathrm{~K}$ (black).

The variability of the arc shape manifests in the calculated voltage (the peak value of the electric potential field at the arc root surface on the electrode) as shown in Figure 3(a). Taking a discrete Fourier transform of the voltage data for the last $5 \mathrm{~ms}$ using the fast Fourier transform (FFT) algorithm [7] gives the result shown in Figure 3(b) - the gradient of the falloff in the frequency range between $5 \mathrm{kHz}$ and $100 \mathrm{kHz}$ is seen to be in excess of 2 decades per decade, indicating the strong presence of high-frequency inertial components in the model arc behaviour. 


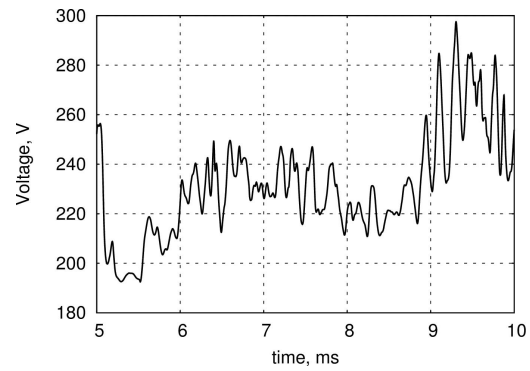

(a)

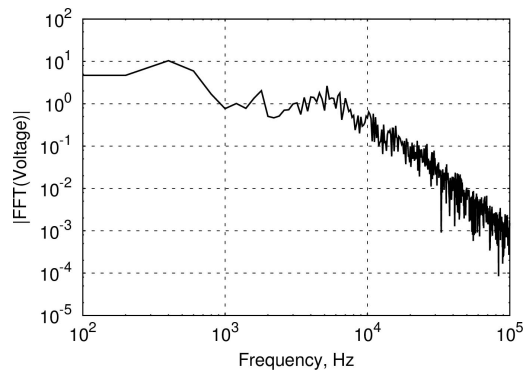

(b)

Figure 3: Voltage data generated from computational arc model,

(a) time series, (b) Fourier transform magnitude spectrum.

\section{Experimental equipment}

A prototype DC arc detection instrument was constructed and used for measurement of the electrical variables on various pilot plant furnaces and test facilities at Mintek. High sampling rates (up to $30 \mathrm{kHz}$ ) were used in order to accurately measure fluctuations induced by the arc in the electrical circuit. Some photographs of the prototype unit are shown in Figure 4.

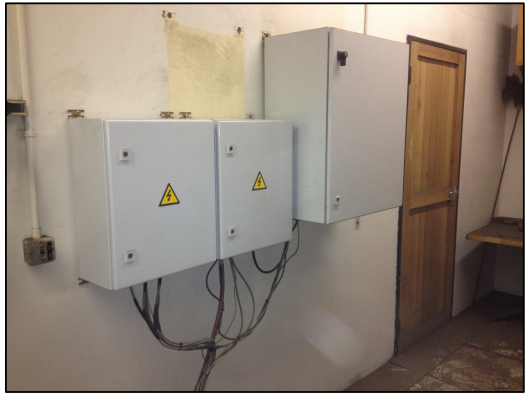

(a)

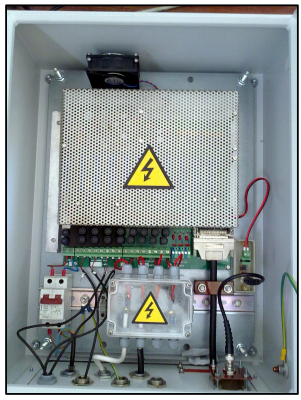

(b)

Figure 4: Photographs of arc detection instrument prototype.

In Figure 4(a), the complete installation is shown. The two boxes at left contain signal conditioning hardware and connect directly to the busbars exiting the DC furnace power supply units located in the room below. The larger box at right contains a desktop computer with a Data Translation data acquisition card capable of logging measurements at the required rate of 30 $\mathrm{kHz}$. The computer also includes customised software for the purpose of computing and analysing the frequency spectrum of the signal using fast Fourier transform (FFT) methods.

Figure 4(b) shows detail of the voltage signal conditioning hardware. At top is an Arcmon [8,9] signal conditioning and isolating board, which prepares the raw voltage signal for input to the 
data acquisition system. The smaller box at bottom contains surge arrestor circuitry. This is designed to limit the peak voltage that is passed through to the sensitive Arcmon circuit board from the voltage measurement input. The open-circuit voltage for rectifiers with large inductances in series can rise significantly higher than the normal operating voltages for short periods of time; the surge arrestor hardware safely steps down the voltage before it enters the Arcmon board, and redirects any extreme spikes to ground.

Testing of the arc detection prototype was accomplished using two pilot-plant furnace facilities at Mintek. For the first series of tests, a 3.2 MVA power supply using an IGBT (Insulated-Gate Bipolar Transistor) high-frequency switching DC rectifier was used to generate arcs in the air gap between a $200 \mathrm{~mm}$ pre-baked graphite electrode and a flat graphite block connected to the anode. No furnace shell or roof was present for these tests, enabling the arc to be observed directly. An Olympus iSpeed 3 high-speed video camera with a Tokina $80-200 \mathrm{~mm}$ f/4 lens was used to photograph the arc during these tests. The Olympus camera and the arc detection prototype were triggered simultaneously using a signal splitter, enabling the electrical and visual measurements to be synchronized and compared.

For the second series of tests, a 5.6 MVA power supply using a 12-pulse thyristor DC rectifier was used to power a test furnace $4.25 \mathrm{~m}$ in diameter smelting an iron and nickel-bearing raw material. The position of the electrode relative to the slag surface was determined by visual inspection, and tests using the arc detection prototype were conducted with the electrode tip positioned both above and below the slag-gas interface.

\section{Test results and discussion}

\section{IGBT power supply - arc in air}

For this test, the prototype arc detection unit was connected to the 3.2 MVA IGBT-type DC power supply. The facility was configured with a single $200 \mathrm{~mm}$ diameter graphite electrode mounted above a $500 \mathrm{~mm} \times 500 \mathrm{~mm} \times 500 \mathrm{~mm}$ graphite block connected to the anode. The arc was struck and operated in the air gap between the electrode and the graphite block. The presence of an arc was confirmed visually and photographically for each of the tests. The arc detection prototype's sample rate was set at $15 \mathrm{kHz}$. Sample sets of 2 seconds in length were obtained, portions of which were used for the FFT analysis in each test.

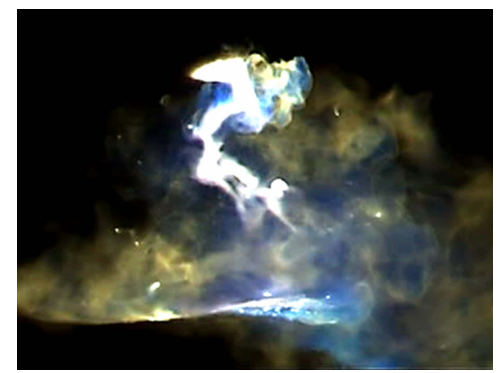

(a) $-0.710 \mathrm{~s}$

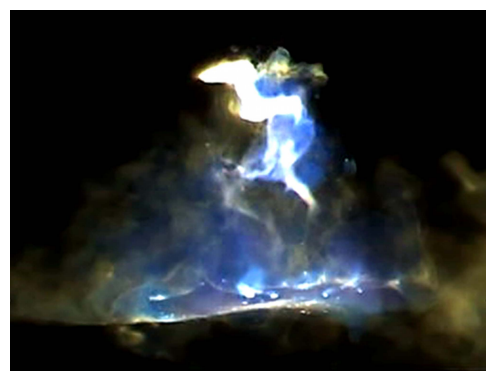

(b) $-0.552 \mathrm{~s}$

Figure 5: Images from high-speed video of arc jet, shutter speed $5 \mu \mathrm{s}$. Times relative to trigger point. 
Test 1 was conducted at $3 \mathrm{kA}$ current and an arc length of approximately $15 \mathrm{~cm}$. Some images of the turbulent arc jet are shown in Figure 5.

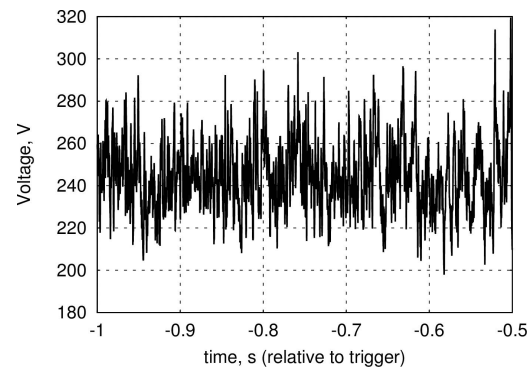

(a)

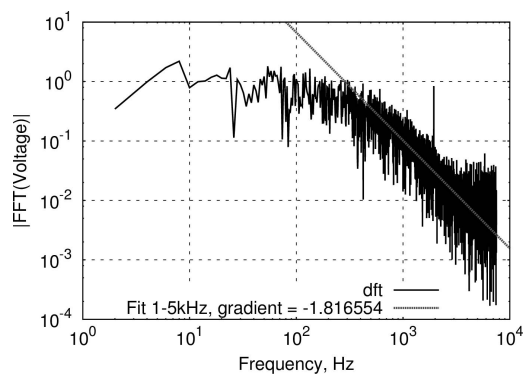

(b)

Figure 6: Test 1 results, (a) time series of measured arc voltage,

(b) the Fourier transform magnitude spectrum.

The measured voltage data for the test are shown in Figure 6. Figure 6(a) shows the time series, while Figure 6(b) shows the Fourier spectrum together with the fitted gradient in the power-law falloff region between 1 and $5 \mathrm{kHz}$.

An additional test was conducted at $1.5 \mathrm{kA}$ current and $35 \mathrm{~cm}$ arc length. The results from both tests are shown in Table II.

Table II: Results of IGBT tests, arc in air.

\begin{tabular}{|c|c|c|c|c|}
\hline Test ID & Arc present? & Current & Arc length & Falloff, decade/decade \\
\hline Test 1 & Yes & $3.0 \mathrm{kA}$ & $15 \mathrm{~cm}$ & -1.82 \\
\hline Test 2 & Yes & $1.5 \mathrm{kA}$ & $35 \mathrm{~cm}$ & -1.90 \\
\hline
\end{tabular}

Both tests at different currents and arc lengths yielded a falloff gradient of nearly -2 decades per decade in the high-frequency region between 1 and $5 \mathrm{kHz}$. This behaviour correlates well with the arc theory and model examined earlier.

\section{Thyristor power supply - arc in furnace conditions}

A second series of tests was conducted on Mintek's Bay 2 smelter pilot-plant during normal operation. This facility uses a 5.6 MVA Thyristor-type DC power supply to operate a DC arc furnace. $200 \mathrm{~mm}$ prebaked graphite electrodes are used, and the furnace anode features a multiple-pin design. The process operates with both a slag and metal pool, ensuring optimal conditions for testing the arc detection principle.

During these tests, the hydraulic electrode hoist was set to manual control. An electrode touchdown (locating the tip of the electrode at the surface of the molten slag) was performed giving a hoist zero position. A visual inspection was then performed to confirm that the tip of the electrode was touching the surface of the slag bath. With this reference point, arc length or electrode immersion depth could be calculated using the position of the hoist at any given time. The arc detection prototype's sample rate was set at $30 \mathrm{kHz}$. Measurements of furnace voltage 
were obtained at between 4 and $7 \mathrm{kA}$ current, using arc lengths and electrode immersions up to $15 \mathrm{~cm}$. Up to five sample sets, each 2 seconds long, were obtained during each test. The full sample length was used for FFT analysis.

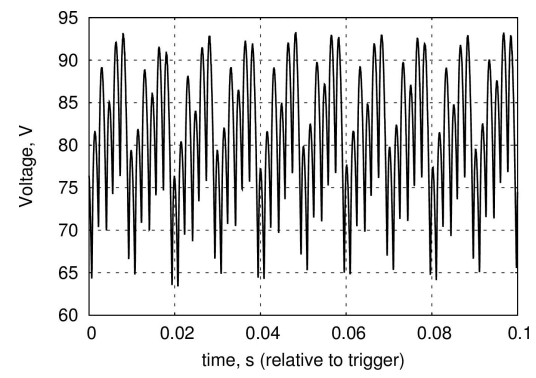

(a)

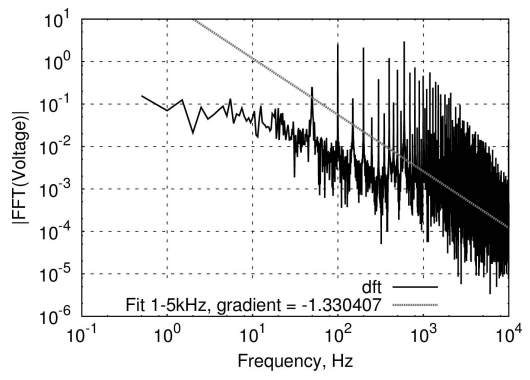

(b)

Figure 7: Test results at $4 \mathrm{kA}$ current and $11 \mathrm{~cm}$ electrode immersion, (a) a section of the voltage time series, (b) the Fourier transform magnitude spectrum.

The time series and Fourier spectra for example tests with the electrode immersed in the slag (no arc present) and the electrode above the slag (arc present) are shown in Figures 7 and 8 respectively.

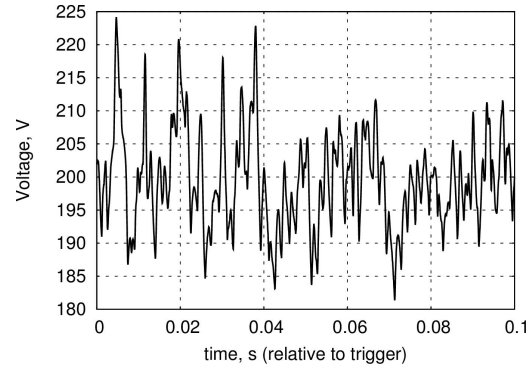

(a)

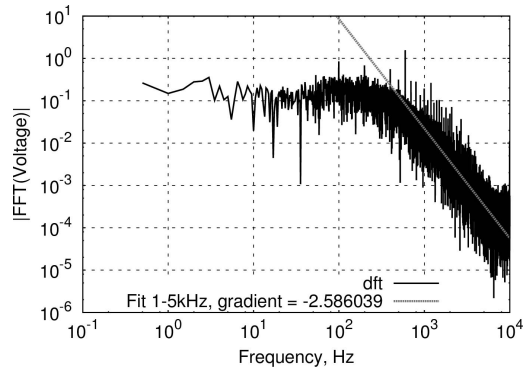

(b)

Figure 8: Test results at $4 \mathrm{kA}$ current and $10 \mathrm{~cm}$ arc length, (a) a section of the voltage time series, (b) the Fourier transform magnitude spectrum.

Results from all tests on the 5.6 MVA facility are collected in Table III. Negative arc lengths indicate immersion of the electrode in the slag, with the furnace operating in resistance-heating mode with no arc present.

For all test cases in which the furnace was operated with the electrode submerged in the slag to some degree, the falloff in the $1-5 \mathrm{kHz}$ range exhibited a shallower slope. As soon as an arc was present in the circuit the shape of the Fourier spectrum changed noticeably, with a sharp knee point arising in the data around $1 \mathrm{kHz}$. This is as a result of a significant increase in the high-frequency falloff values, with all open-arc tests under furnace conditions yielding a steeper 
slope; this is consistent with the presence of a dynamic arc exhibiting an inertial response as discussed earlier.

Table III: Results of Thyristor tests, arc in furnace conditions.

\begin{tabular}{|c|c|c|c|c|c|}
\hline Test ID & Arc present? & Current & Arc length & $\begin{array}{c}\text { Falloff mean, } \\
\text { decade/decade }\end{array}$ & $\begin{array}{c}\text { Falloff variance s }^{2} \text {, } \\
\text { decade }^{2} \text { decade }^{2}\end{array}$ \\
\hline Test 1 & Yes & $4.0 \mathrm{kA}$ & $10 \mathrm{~cm}$ & -2.62 & 0.00229 \\
\hline Test 2 & No & $4.0 \mathrm{kA}$ & $-6 \mathrm{~cm}$ & -1.41 & 0.00300 \\
\hline Test 3 & No & $4.0 \mathrm{kA}$ & $-11 \mathrm{~cm}$ & -1.31 & 0.01415 \\
\hline Test 4 & No & $4.0 \mathrm{kA}$ & $-16 \mathrm{~cm}$ & -1.41 & 0.00301 \\
\hline Test 5 & Yes & $4.0 \mathrm{kA}$ & $10 \mathrm{~cm}$ & -2.51 & 0.03154 \\
\hline Test 6 & Yes & $6.8 \mathrm{kA}$ & $10 \mathrm{~cm}$ & -2.15 & 0.00121 \\
\hline
\end{tabular}

\section{Conclusions}

Development and application of a prototype instrument for the problem of measuring the presence of a plasma arc in a DC furnace has been largely successful.

Study of the theory of plasma arc dynamics suggested a practical measurement technique based on Fourier analysis that may be used to discern between open-arc and submerged operating conditions.

The arc detection prototype was successfully operated on various pilot-scale DC power supply and DC furnace facilities. Data gathered during these tests supported the theory of the arc as an inertial response system, with a characteristic Fourier spectrum falloff of approximately -2 decades per decade or lower observed in the voltage signal at high frequencies when an arc was present in the circuit.

It would therefore appear that the decade per decade falloff in the high-frequency $1-5 \mathrm{kHz}$ range is a suitable indicator for the presence of an arc, at least at pilot-plant scale. The identified process for implementing an arc detection device based on this technique is as follows:

- Measure the DC voltage signal between the furnace anode and cathode connections at high sample rates, in excess of $15-30 \mathrm{kHz}$

- Perform FFT analysis on sections of the measured signal

- If certain threshold criteria are met, issue a signal indicating that the furnace is operating with an open arc

Such an instrument may be usefully applied as a control or operator-guidance tool on DC plasma arc furnaces used for any of a variety of applications, especially those in which the presence of an arc is necessary but difficult to verify. 


\section{Acknowledgements}

This paper is published by permission of Mintek. Access to high-performance computing facilities at the Centre for High Performance Computing in Cape Town, South Africa was invaluable for performing model arc calculations.

\section{References}

1. R.T. Jones and T.R. Curr. "Pyrometallurgy at Mintek", Proceedings of Southern African Pyrometallurgy 2006 , ed. R.T. Jones (Johannesburg, South Africa: Southern African Institute of Mining and Metallurgy, 2006), 127-150.

2. Q.G. Reynolds and R.T. Jones, "Semi-empirical modelling of the electrical behaviour of DCarc smelting furnaces", Journal of the South African Institute of Mining and Metallurgy, 7 (2004), 345-351.

3. C. Walker, K. Sutherland, B. Marshall, J.M.A. Geldenhuis, J. Els, N. Voermann, and B. Wasmund. "Conversion to Partially Open Bath Smelting on Highveld Ironmaking Furnaces", Proceedings of the European Metallurgical Conference 2007, ed. GDMB Gesellschaft der Metallurgen und Bergleute e.V. (Clausthal-Zellerfeld, Germany: GDMB Gesellschaft der Metallurgen und Bergleute e.V., 2007) 895-914.

4. Q.G. Reynolds, "The dual-electrode DC arc furnace - modelling brush arc conditions", Journal of the Southern African Institute of Mining and Metallurgy, 7 (2012), 605-611.

5. Q.G. Reynolds, R.T. Jones and B.D. Reddy. "Mathematical and computational modelling of the dynamic behaviour of direct-current plasma arcs", Proceedings of the 12th International Ferroalloys Congress (INFACON XII), ed. A. Vartiainen (Espoo, Finland: Outotec Oyj, 2010), 789-801.

6. B. Bowman. "Properties of arcs in DC furnaces", Proceedings of the 52nd Electric Furnace Conference, ed. Iron and Steel Society (Nashville TN, USA: Iron and Steel Society, 1994), 111120.

7. FFTW, http://www.fftw.org.

8. I.J. Barker, M.S. Rennie, C.J. Hockaday and P.J. Brereton-Stiles. "Modes of electrical conduction in industrial silicon-type furnaces", Proceedings of Silicon for the Chemical Industry VIII, ed. H.A. Oye, H. Brekken, T. Foosnes and L. Nygaard (Trondheim, Norway: Norwegian University of Science and Technology (NTNU), 2006), 79-90.

9. I.J. Barker, M.S. Rennie, C.J. Hockaday and P.J. Brereton-Stiles. "Measurement and Control of Arcing in a Submerged-Arc Furnace", Proceedings of the 11th International Ferroalloys Congress (INFACON XI), ed. R.K. Das and T.S. Sundaresan (Mumbai, India: The Indian Ferro Alloys Producers Association, 2007), 685-694. 


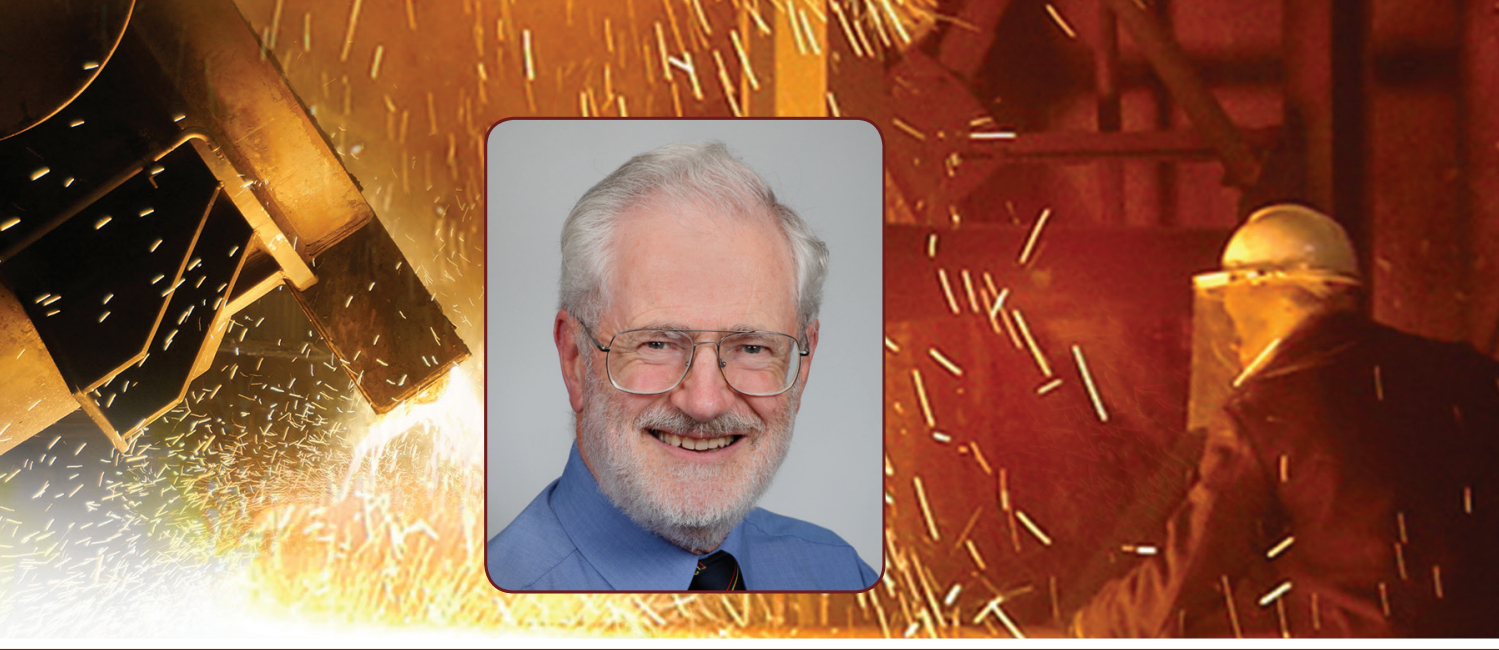

\section{GELEBRATING THE

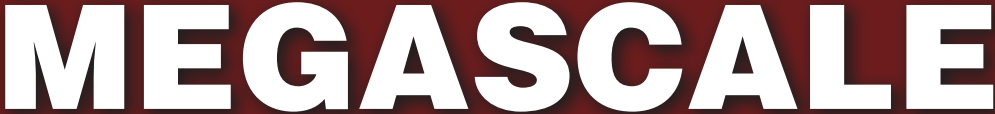

Proceedings of the Extraction and Processing

Division Symposium on Pyrometallurgy in Honor of David G.C. Robertson

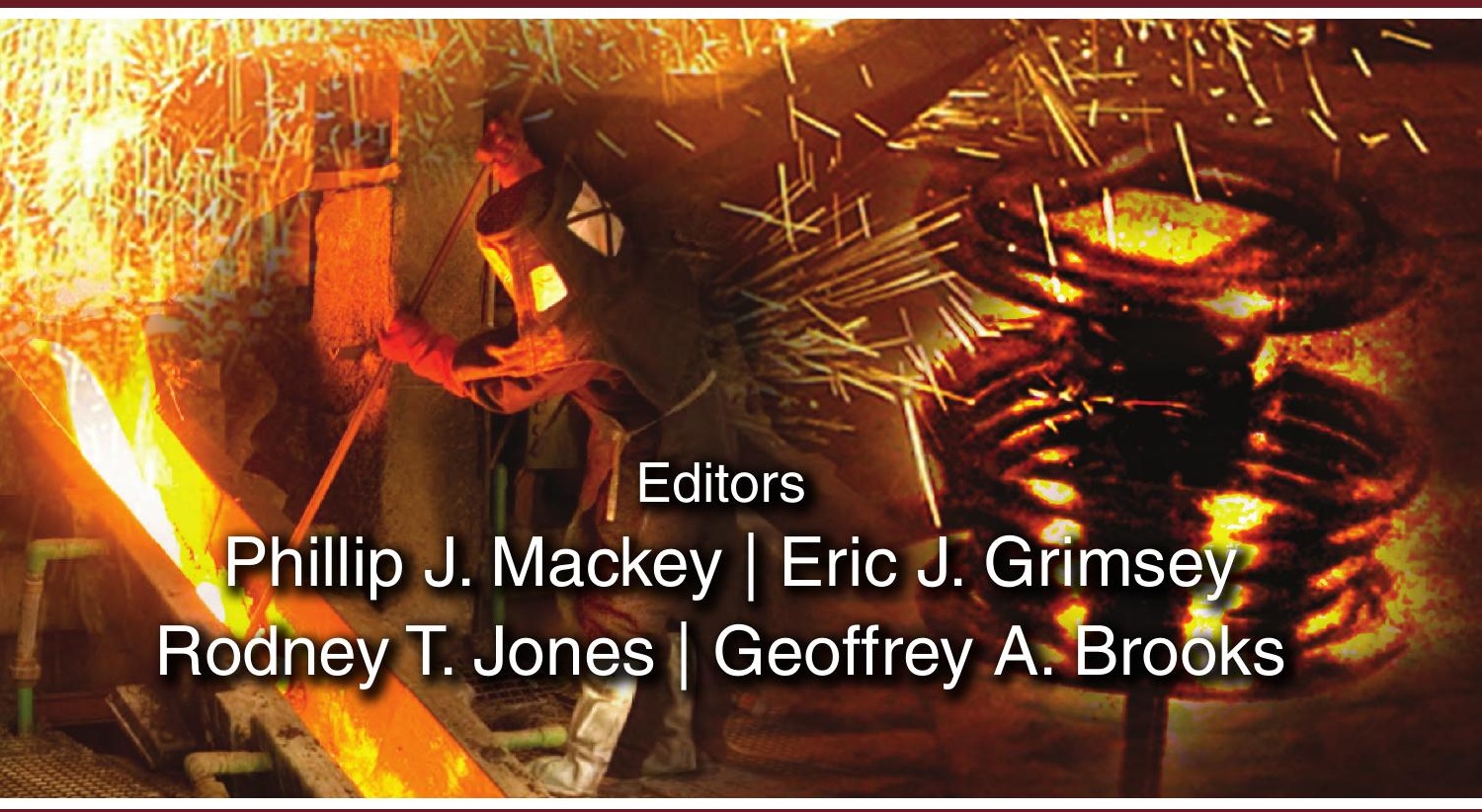

